\title{
Seguimiento fisioterápico a lo largo de 14 meses a niño con síndrome de Sandifer
}

\author{
A 14-month physiotherapy follow up to a child with \\ Sandifer syndrome
}

\author{
M. A. Capó-Juan¹ y M. A. Marquès-Castell²
}

${ }^{1}$ Área de Fisioterapia. Departamento de Enfermería y Fisioterapia. Universidad de las Islas Baleares, Palma, España. ${ }^{2}$ Servei de Desenvolupament Infantil i Atenció Primerenca. Fundació per a persones amb discapacitat de Menorca, Islas Baleares, España

\section{ABSTRACT}

Introduction: The Sandifer's Syndrome was described in 1964 by the neurologist Paul Sandifer when detected a disorder of the upper gastrointestinal tract that generated gastroesophageal reflux disease, especially in new-borns and children. It tends to present with esophagitis, iron deficiency anemia and paroxysmal postural movements. These symptoms are related with the ingestion and they use to remit during the sleep. This syndrome generates to the child a psychomotor delay and, therefore, the Early Care is essential to detect and to identify all the signs early; always focusing on the process of learning and consolidation of psychomotor achievements. Thus, the treatment consists of stimulating the child trough the active game to get the psychomotor progression, working on perceptual and sensory changes, and avoiding pain during the intervention.

Case report: 8-month-old lactating child with psycmotor delay referred to Early Care from Hospital. The baby presents neck hyperextension, cervicoaxial hypotonia, rejection of the prone position, opisthotonus in supine position and sporadic regurgitations. The child can not move neither maintains the sedestation. Furthermore, he does not hit toys; he has good contact but does not point out objects and does not emit bisyllables.

Comments: After fourteen months of Early Care (Physiotherapy), the toddler can up and down the stairs grabbed to the railing with one hand and he gets up from the ground practically alone. He can make more complex lace with dominance of the right hand. The toddler understands concepts of permanence and of

Capó-Juan MA, Marquès-Castell MA. Seguimiento fisioterápico a lo largo de 14 meses a niño con sindrome de Sandifer. Rev Soc Esp Dolor. 2020;27(5):329-331

\section{RESUMEN}

Introducción: El síndrome de Sandifer fue descrito en 1964 por el neurólogo Paul Sandifer cuando detectó un trastorno del tracto gastrointestinal superior, que generaba enfermedad de reflujo gastroesofágico, especialmente en bebés y recién nacidos. Suele presentarse con esofagitis, anemia ferropénica y movimientos posturales paroxísticos relacionados con la ingesta, que remiten durante el sueño. Puede provocar en el niño un retraso psicomotor y, por lo tanto, la detección y la identificación precoz de los signos son fundamentales. Con respeto al tratamiento no farmacológico, la estimulación precoz resulta imprescindible en el proceso de aprendizaje y la consolidación de hitos psicomotrices. El tratamiento consiste en estimular al niño mediante el juego activo para la progresión psicomotora y trabajar las alteraciones perceptivas y sensoriales evitando el dolor.

Caso clínico: Lactante de ocho meses con retraso psicomotor derivado a Atención Temprana desde el ámbito hospitalario. Presenta hiperextensión de cuello, hipotonía cervicoaxial, rechazo del decúbito prono, opistótonos en decúbito supino y regurgitaciones esporádicas. A nivel motriz no tiene ninguna vía de desplazamiento y tampoco mantiene la sedestación; en cuanto a la manipulación, aún no choca juguetes. A nivel comunicativo, el contacto es bueno, pero no señala objetos ni emite bisílabos.

Comentarios: Después de catorce meses de Atención Temprana (Fisioterapia), el niño sube y baja escaleras cogido de la barandilla con una mano y puede

Recibido: 02-05-2020

Aceptado: 10-09-2020

Correspondencia: Maria A. Marquès Castell mariamarquescastell57@gmail.com 
action-reaction; and he repeats many words and emits more than ten words spontaneously. The family collaboration by applying the recommended patterns has been a key piece in the treatment. The guidelines applied in various settings facilitated the achievement of motor skills and the consolidation of stages.

Key words: Sandifer's syndrome, gastroesophageal reflux, psychomotor performance, pain, early intervention. levantarse del suelo casi solo. Además, puede realizar encajes complejos, con dominancia de la mano derecha. A nivel cognitivo-comunicativo comprende los conceptos de permanencia y de acción-reacción, repite muchas palabras y emite de forma espontánea más de diez palabras. La colaboración familiar aplicando las pautas recomendadas ha sido una pieza clave en el tratamiento. Así, las pautas aplicadas en diversos entornos facilitaron la consecución de hitos motrices y el afianzamiento de etapas.

Palabras clave: Síndrome de Sandifer, reflujo gastroesofágico, desempeño psicomotor, atención temprana, dolor.

\section{INTRODUCCIÓN}

El síndrome de Sandifer (SS) fue descrito en 1964 por el neurólogo Paul Sandifer, detectando un trastorno del tracto gastrointestinal superior que generaba enfermedad de reflujo gastroesofágico (ERGE), especialmente en bebés y recién nacidos (1). Se caracteriza por esofagitis, anemia ferropénica, movimientos en hiperextensión de cuello, cabeza y tronco, así como rotación de la cabeza durante o justo después de la ingesta, que remiten durante el sueño [2]. Así pues, el reflejo extensor y la posición de la cabeza son una respuesta del niño que lleva a disminuir el dolor abdominal causado por el SS (3).

En cuanto al tratamiento, la supresión ácida con un bloqueante $\mathrm{H}_{2} \mathrm{O}$ un inhibidor de la bomba de protones pueden ayudar al niño con ERGE (4). Resulta importante no confundir el SS con el cólico del lactante u otros problemas digestivos al evaluar a un niño con tortícolis o una postura inusual que no está asociada con una enfermedad o lesión neuromuscular (5).

EI SS puede repercutir en el niño generando un retraso psicomotor, por lo que la detección y la identificación precoz de este retraso son fundamentales para su bienestar y su entorno [6]. La estimulación precoz de las carencias en el desarrollo psicomotor será clave para la inhibición de los patrones inadecuados adquiridos. El tratamiento aplicado consiste en estimular el niño mediante el juego activo para conseguir la progresión psicomotora más adecuada, evitando el dolor y malestar generado por alteraciones perceptivo-sensoriales y por el propio SS [7].

\section{CASO CLÍNICO}

Lactante de ocho meses con retraso psicomotor derivado a Atención Temprana desde el ámbito hospitalario.

Sin antecedentes familiares graves, embarazo controlado a término con parto eutócico de 41 SG; sin signos de complicación, plagiocefalia ni presentación con doble vuelta de cordón en el parto; APGAR 9/10; perímetro craneal $36 \mathrm{~cm}$; examen neonatal y test de metabulopatías normales. El motivo de la primera consulta a los dos meses fue una hiperextensión del cuello, sobre todo al cogerlo en brazos (pull to sit), hipotonía cervicoaxial, rechazo del decúbito prono, opistótonos en decúbito supino y regurgitaciones esporádicas. Por eso, se solicitó una ECO cerebral y, posteriormente, a los tres meses, se detectó una tortícolis paroxística benigna del lactante. Asimismo, se programó una pHmetría.

La exploración neurológica a los cuatro meses no detectó signos anormales (pares craneales, tono y reflejos correctos; cabe resaltar que, por lo que hace a la progresión de los reflejos primitivos hacia otros tipos de reflejos, nos llama la atención que los reflejos primitivos estuvieran presentes tanto tiempo en el niño); asimismo, no se tienen datos sobre la exploración física del bebé que pudieran ayudar a orientar el tratamiento manual. Los resultados de la ECO cerebral, EEG, ECG, RM craneal y de columna fueron normales. Pruebas complementarias: pHmetría con valores dentro de la normalidad pero con límites altos.

Los estudios inmunológicos solicitados a los siete meses determinaron la intolerancia a la ingesta de proteínas de leche de vaca.

Respeto a la intervención, y previamente al inicio de la atención temprana, empezó la estimulación fisioterápica a los cuatro meses en el ámbito hospitalario. La valoración por parte del fisioterapeuta de los servicios de atención temprana a los nueve meses de edad indicó: hipertonía, gran reflejo extensor, opistótonos, pull to sit y control cefálico pobres, dificultad para la sedestación y rastreo casi ausente. El objetivo inicial planteado en las sesiones fue inhibir los hábitos posturales y los movimientos que interferían en la motricidad normal, además de controlar el reflejo extensor y conseguir una sedestación autónoma. De esta manera, no se siguió ningún método específico de rehabilitación neurofuncional para la mejora del desarrollo psicomotriz del niño, más allá de facilitar dichas etapas de desarrollo respetando sus tiempos y conseguir posiciones funcionales mediante el juego. Al primer mes de terapia se consiguió una mejora significativa del pull to sit con excelente control cefálico. A los diez meses el niño consiguió la sedestación mantenida durante cinco minutos, y a los diez y medio el rastreo. A partir de los once meses 
se propició la posición cuadrúpeda y la promoción del desplazamiento mediante el gateo, promoviendo la participación y la coordinación de los cuatro miembros. Para facilitar dicho hito, el entrenamiento del gateo se realizó en rampa (inclinación de $45^{\circ}$ ). A los trece meses logró el gateo coordinado y simétrico. Un mes después se facilitó el trabajo de rodilla y la posición de caballero, que condujo al mantenimiento de la bipedestación estática y a la marcha lateral, lograda a los catorce meses. Al año y medio, el niño adquirió un mejor control motriz y una mayor consciencia corporal.

A los veinte meses inició los primeros pasos solo, pero con falta de confianza y ligera inestabilidad con escasas reacciones de equilibrio caminando con guardia alta. A los veintiún meses consiguió la marcha autónoma; y a los veintidós pudo subir y bajar escaleras cogido de la barandilla con una mano, aunque a veces para bajar la escalera solía rotar el tronco y realizar marcha lateral. Para levantarse del suelo tendía a buscar un punto de soporte, pero no siempre.

A nivel motor fino, cabe señalar que el niño empezó a chocar juguetes entre ellos y sostenerlos durante cinco minutos en sedestación a los diez meses; a apilar dos bloques a los doce; a encajar el círculo a los trece, y el triángulo y el cuadrado a los catorce. A los veintidós meses podía realizar encajes más complejos y hacer coordinación óculo-manual con dominancia de la mano derecha. Además, inició la prensión de la lapicera de forma trifalángica distal para empezar el garabateo.

A nivel cognitivo, comprendió los conceptos de permanencia y de acción-reacción; e inició el juego imitativo al año y el simbólico a partir de los veinte meses.

A nivel comunicativo, a los trece meses empezó a señalar objetos, a pronunciar bisílabos repetitivos ("mama"] y a despedirse con la mano. A los veintidós meses ya emitía veinte palabras y repetía las terminaciones de muchas de ellas.

En cuanto a la estimulación del desarrollo psicomotor, las herramientas terapéuticas se basaron en las prácticas centradas en la familia [8]. Así, las pautas aplicadas en diversos entornos facilitaron el logro de hitos motrices y el afianzamiento de etapas, siempre respondiendo a las preocupaciones y objetivos de la familia.

Para disminuir el dolor, el malestar y los patrones postulares disfuncionales se dieron pautas a lo largo del proceso de intervención como: 1] favorecer posturas que promovieran el desarrollo psicomotor, promoviendo en las primeras fases el decúbito prono y posiciones funcionales que evitaran la hiperextensión de cuello y la tortícolis; 2) aplicar algunas recomendaciones fisioterápicas de terapia manual y visceral utilizadas en el cólico del lactante en las primeras fases; y 3] favorecer la lactancia materna y ayudar en la transición al proceso de alimentación [9]. El juego supuso la clave dentro de la terapia por parte del fisioterapeuta, proponiendo diversas actividades. Además se recibió asesoramiento por parte del psicólogo de atención temprana para determinadas conductas.

\section{DISCUSIÓN}

En los años cincuenta se mantenía la idea de que los lactantes con RGE, mantenidos sentados día y noche inclinados entre $45-60^{\circ}$ de forma repetitiva y constante hasta seis-ocho meses, dejaban de producir vómitos (10). Hoy en día, ha cambiado la visión, incluyendo la atención temprana liderada por fisioterapia como intervención fundamental. Así, una derivación a atención temprana en edad muy precoz podría evitar un tratamiento tan prolongado a lo largo del tiempo, siendo además fundamentales para la intervención las prácticas centradas en la familia [8].

El caso permite mostrar la influencia que puede tener una alteración digestiva sobre el desarrollo psicomotor, y se destaca la figura del fisioterapeuta como referente terapéutico (11).

\section{CONFLICTO DE INTERESES}

Ningún conflicto de interés. No se obtuvieron becas para la realización de este estudio.

\section{BIBLIOGRAFÍA}

1. Bamji N, Berezin S, Bostwick H, Marvin S, Medow MD. Treatment of Sandifer Syndrome with an Amino-Acid-Based Formula. AJP Rep. 2015;5(1):e51-e52. DOI: 10.1055/s0035-1545672

2. Quintero Ml, López K, Belandria K, Navarro D. Síndrome de Sandifer. A propósito de la enfermedad por reflujo gastroesofágico en niños. Rev Gen. 2012;66(2):133-5.

3. Narchi H. A Rare Under-Recognized Cause of Pseudo-Seizures. International Pediatrics. 2004;19(1):39-41.

4. Cochran WJ. Reflujo gastroesofágico en lactantes. Manual MSD; 2020 [Consultado el 15 de junio de 2018]: Disponible en: https://www.msdmanuals.com/ es/professional/pediatr\%C3\%ADa/trastornos-gastrointestinales-en-reci\%C3\%A9n-nacidos-y-lactantes/reflujogastroesof\%C3\%A1 gico-en-lactantes

5. Moore DM, Rizzolo D. Sandifer syndrome. JAAPA. 2018;31(4):18-22. DOI: 10.1097/01.JAA.0000531044. 72598.26.

6. Council on Children With Disabilities; Section on Developmental Behavioral Pediatrics; Bright Futures Steering Committee; Medical Home Initiatives for Children With Special Needs Project Advisory Committee. Identifying infants and young children with developmental disordersin the medical home: an algorithm for developmental surveillance and screening. Pediatrics. 2006;118(1):405-20. DOI: 10.1542/ peds.2006-1231

7. Capó-Juan MA, Capó-Juan MM. Preescolar de 13 mesos intolerant als canvis posturals. Pediatr Catalana. 2016; 76(4):149-51.

8. Dalmau M, Balcells-Balcells A, Giné C, Cañadas M, Casas O, Salat $Y$, et al. How to implement the family-centered model in early intervention. Anal Psicol. 2017;33(3):641-51. DOI: 10.6018/analesps.33.3.263611.

9. García-Marqués S, Chillón-Martínez R, González-Zapata S, Rebollo-Salas M, Jiménez-Rejano JJ. Efectividad de la terapia manual visceral en el tratamiento del cólico del lactante. Cuest Fisioter. 2017;46(2):105-12.

10. Casasa JM. El reflux gastroesofàgic i la medicina basada en l'evidència. Pediatr Catalana. 2001;61:269-78.

11. Capó-Juan MA. Efectividad de programas educativo-terapéuticos en Fisioterapia. Rev Soc Esp Dolor. 2016;23[3]:154-8. 